\title{
A Study of Textural Analysis Methods for the Diagnosis of Liver Diseases from Abdominal Computed Tomography
}

\author{
Gunasundari S \\ Research Scholar \\ Pondicherry University \\ Puducherry
}

\author{
Janakiraman S \\ Asst Prof \\ Pondicherry University \\ Puducherry
}

\begin{abstract}
Liver diseases are considered seriously because liver is a vital organ to human beings. Computer aided liver analysis is a technique that can help radiologists to accurately identify diseases that can help in reducing the risk of liver surgery. The computer aided diagnosis (CAD) system consists of the segmentation of liver and lesion, extraction of features from a lesion and characterization of liver diseases by means of a classifier. In the last decade, the use of many segmentation techniques and classifier systems have been proposed by many authors with the intention to increase the performance of CAD systems This article focuses on various textural analysis methods used so far for the classification of liver diseases from abdominal Computed Tomography scans. It reviews the techniques and results of the various methods are analyzed and summarized. The future direction for the research is also discussed
\end{abstract}

\section{General Terms}

Pattern Recognition, Survey

\section{Keywords}

CT, Textural Features, Neural Network, Co occurrence Matrix, CAD, Feature Selection, Liver

\section{INTRODUCTION}

The most familiar medical imaging studies for early identification of liver diseases comprise ultrasonography (US), computed tomography (CT), magnetic resonance imaging (MRI). CT is often the preferred method for diagnosing many different cancers than ultrasonography, since the image allows a physician to confirm the presence of a tumor and to measure its size, precise location and the extent of the tumor's involvement with other nearby tissue. Despite the excellence of CT images has been appreciably improved during the last years, it is hard in some cases, even for experienced doctors, to make a $100 \%$ precise diagnosis. In radiology computer-aided diagnosis (CAD) are procedures in medicine that help doctors in the analysis of medical images. CAD system can only provide a second opinion and cannot replace radiologists.

The Liver CAD System consists of Preprocessing, Segmentation of Liver, ROI Analyze and Classification. Preprocessing is to decrease of artifacts, image noise reduction and leveling of image quality. The liver is positioned on the superior part of the CT abdominal image and takes up the major region in a CT image. Segmentation means extracting the liver from abdominal CT and also extracting tumor. In ROI (Region of Interest) analyze the detected region is analyzed individually to extract textural features. Classification applies any classifier to identify the liver disease based on textural features. Liver cancer is the third most common cancer in the world. A few of these are benign (noncancerous), and some are cancerous and can extend to further parts of the body. The most common benign tumors of the liver include Hemangioma, Hepatic adenoma, and Focal nodular hyperplasia, Cysts, Lipoma, Fibroma and Leiomyoma. They may require to be removed surgically if they cause ache or hemorrhage. Liver cancers include Hepatocellular carcinoma (HCC) and Cholangiocarcinoma. Texture is a frequently used characteristic in the analysis and understanding of images. The statistical properties of pixel gray level intensity are used to characterize the texture. Usually, texture can be expressed by statistical, structural or spectral techniques such as transforms, run-length matrices, spectral measures, Fractal dimensions, statistical moments, auto covariance and co-occurrence matrices. This paper is organized as follows. Section II presents the review of various textural features and classifier. Section III describes methods for the classification of liver diseases from abdominal CT. Section IV summarizes the textural analysis techniques. Finally, a brief conclusion and future work are included in Section V.

\section{TEXTURAL FEATURES AND CLASSIFIER}

A statistical approach perceives a texture as a quantitative measure of the arrangement of intensities in an area and it is more extensively used. Some extensively used techniques for feature extraction are Gray Level Difference Statistics (GLDS), Spatial Gray level Dependence Matrices (SGLDM), Gray level Run length matrix (GLRLM), Gray Level Histogram, Laws Texture Energy Measure (TEM), Wavelet Features. The texture features are mean, standard deviation, variance, kurtosis, skewness, Inverse Difference Moment, maximum probability, Contrast, Cluster Shade, Cluster Prominence, Uniformity, Entropy, Information about Correlation, Homogeneity, Angular Second Moment, Dissimilarity, run length, Fractal Dimension Measurement (FDM) etc.

An overview of algorithms for statistical texture analysis of 2D images is given [1]. Statistical methods can be categorized into first order (one pixel), second-order (two pixels) and higher-order (three or more pixels) and spectral statistics, based on the number of pixels used to define the feature. First order statistics (FOS) are calculated from the original image values. First order features include moments such as mean, variance, dispersion, average energy, entropy, skewness and kurtosis. Spatial gray level co-occurrence calculates image properties associated to second-order statistics which think about the relationship between pixels (usually two). The gray level co-occurrence matrices (GLCM) are recommended and it is based on the joint probability distributions of pairs of pixels [2]. Another widely used 
feature is Gray Level Difference Statistics (GLDS). GLDS is the Probability Density Function (PDF) of pair pixels lying at a specific distance and having a particular intensity value difference. Inter pixel gray level values have a large variety of fine texture and the least variation for coarse texture. The texture transform represent a different approach to texture investigation. The most commonly used are Fourier, Haar, Gabor function and wavelet transforms.

Feature selection selects the subset of features to decrease the feature space which gets better the prediction accuracy and decrease the calculation time. This is accomplished by eliminating inappropriate, unnecessary and noisy features. Different feature ranking and feature selection methods have been projected in the machine learning journalism, Such as Correlation-based Feature Selection (CFS), Principal Component Analysis (PCA), Gain Ratio (GR) attributes evaluation and Chi-square Feature Evaluation. Some of these methods do not perform feature selection but only feature ranking. Forward selection, backward elimination, bi-directional search, best-first search and genetic search methods are frequently used in this job. A number of such procedures have been proposed but popularly used feature selection algorithms are Sequential forward Selection (SFS), Sequential Backward selection (SBS), Genetic Algorithm (GA) and Particle Swarm Optimization.

In CAD based liver disease classification, texture features are identified using co-occurrence matrices, runlength statistics, transforms, Law's texture and statistical moments etc. The textual information obtained from the extracted liver region is used to train the classifier to classify the liver diseases. Clustering algorithm, Support Vector Machine (SVM), neural networks (NN), Minimum Distance Classifier is used as a classifier. Neural networks are perfect in identifying diseases using scans since there is no necessitate providing a specific procedure on how to recognize the disease. The neural network used are Feed forward neural network, Radial basis function (RBF) network, Learning Vector Quantization (LVQ), Back Propagation Network (BPN), Probabilistic Neural Network (PNN), Hopfield network, Cascading neural networks. The advantages of using neural network include Adaptive learning, Self-Organization and Fault Tolerance.

\section{METHODS FOR CLASSIFICATION OF LIVER DISEASE}

A lot of liver texture analysis techniques have been proposed in the past for CT imaging modalities to extract useful features for reliable liver tissue classification. The efforts have been put in surveying the efficiency of textural analysis techniques devised for all imaging modalities [3] whereas our survey consists of only textural techniques applied to abdominal CT image.

\subsection{Support Vector Machine}

Support Vector Machine was designed by some authors to characterize liver diseases using features derived from abdominal CT. The suspicious tumor region is selected manually [4]. Preprocessing adjustments to sub images were used for equalizing the information needed for a differential diagnosis. The auto covariance texture features of sub image were extracted. SVM is used to construct the liver disease diagnosis system for testing the hepatic tumors as benign or malignant. They evaluated 164 liver lesions. Out of 164, 80 are malignant and 84 belong to Hemangioma. The malignant group of tumors includes primary HCC and metastatic tumors. The result shows that a total accuracy rate of $81.7 \%$ is obtained. Kernel-based Classifier is implemented [5] for classification of liver diseases. Liver diseases including cyst, hepatoma and cavernous hemangioma are identified. The ROIs of the normal and abnormal abdominal CT images are extracted then the statistical features are derived from the ROI. SFS algorithm reduces the features space for classification. The multiclass SVM is used to identify the liver disease. In the categorization phase, a 4-layer hierarchical scheme is implemented in the classifier. First layer distinguishes the normal tissue from the abnormal tissues, Second layer classifier discriminate cyst from the other abnormal tissues, Third layer identifies Cavernous hemangioma and hepatoma is recognized from the undefined tissues in the last layer. Receiver Operating Characteristic (ROC) curve is used for evaluating the performance of the diagnosis system. The classifier is evaluated with 146 images of the liver diseases and average AUC of approximately 0.91 was obtained. A multi class SVM classifier is also proposed based on statistical learning theory [6] for automatic classification in liver disease.

ROI is obtained by segmenting the liver from CT images using snake algorithm [7]. The features were extracted using SGLDM. In order to classify liver diseases, the multiclass SVM is used. The first layer was designed to discriminate between diseased and non-diseased tissue, and Second layer differentiates hemangioma from the other Nonhemangioma tissues and the further layer was used to classify it between hepatoma and cirrhosis. An experienced radiologist is used to identify ROI with CT liver images. Texture features based on FOS, SGLDM, GLRLM and GLDM are extracted for each ROI. Multi class SVM is used to classify liver diseases into primary hepatic carcinoma, hemangioma and normal liver [8].

\subsection{Neural Networks}

Neural networks have been extensively used in pattern classification applications. CT liver images are characterized into normal, visible and invisible malignancy [9]. Three different sets of statistical features are used to classify liver images. First set consists of four features namely energy, entropy, local homogeneity and inertia which are computed with SGLDM. Five features specifically short run emphasis, long run emphasis, gray level distribution, run length distribution, run percentages are derived using GLRLM belongs to second set and another five features of GLDM namely contrast, angular second moment, entropy, mean, inverse difference moment comprises the third set. The results show that, except for Local homogeneity (Direction $0^{\circ}$ ) all feature parameters detect early malignancy with a confidence level of above $99 \%$

A CT liver image diagnostic categorization system is developed [10] which consists of a detect-before-extract (DBE) system which finds the liver border without human intervention and a neural network liver classifier for categorization. Classifier uses specially designed feature descriptors to differentiate normal liver, two types of liver tumors, hepatoma and hemangioma. Neural Network is implemented by a modified PNN [MPNN] in combination with feature descriptors which are obtained from Fractal feature information and the gray level co-occurrence matrix. The proposed system was tested with 30 liver cases and revealed to be proficient and very successful. The classification rate obtained is about $83 \%$. A CAD system is presented to discriminate four hepatic tissue types: normal liver, hepatic cyst, hemangioma and HCC from CT images [11]. ROI is drawn by an experienced radiologist on CT images and are given to a feature extraction module. Five different texture feature sets are obtained using FOS, 
SGLDM, GLDM, TEM, and FDM. Then, the full feature sets or their reduced versions after proper GA-based feature selection is combined and fed into five base-level classifiers: one Multilayer Perceptron NN (MLP-NN), one Radial Basis Function NN (RBF-NN), and three k-Nearest Neighbors classifiers. An ensemble of classifiers is constructed by combining the predictions of the base-level classifiers using appropriate voting schemes. The RBF-NN and 1-NN classifiers yielded to worse individual classification performances in the testing set. The best individual classification performance in the testing set was equal to $93.75 \%$ and was achieved by the $\mathrm{k} 1 \mathrm{w}-\mathrm{NN}$ classifier using the reduced feature set. Finally a classification performance of the order of $90.63 \%$ was achieved with voting scheme.

An automatic liver segmentation and classification system is developed. The orthogonal wavelet transform is used to compute horizontal, vertical and diagonal information [12]. Statistical texture features like mean, standard deviation, contrast, entropy, homogeneity and angular second moment are mined from this information. Finally, PNN is used for classification. The proposed system is tested with 100 images of fatty liver and cirrhotic and it produces an accuracy rate of $95 \%$. Work is extended to classify benign and malignant tumor using biorthogonal wavelet transform [13] with LVQ network. Second order statistical texture features such as angular second moment or energy, Contrast, Homogeneity and Entropy in horizontal, vertical and diagonal directions using pixel distance of 1 was calculated. The LVQ neural network is trained using the obtained features. The system is tested with 100 images consists of $34 \mathrm{HCC}, 18$ cholangio carcinoma, 30 hemangioma and 18 adenoma. The classification performance obtained is $92 \%$.

The wavelet and the neural network is evaluated in the differential analysis of liver tumors in CT images [14]. The tumors considered in this study are HCC, cholangio carcinoma, Hemangioma and hepatoadenoma. The tumor was automatically extracted from the CT abdominal images and the textural information found was used to train the PNN to classify the tumors. The optimized feature set was selected by SBS based on the classification performance of PNN. The performance of the method is assessed for the 105 images. It is found that the LVQ neural network produces an accuracy rate of $83.5 \%$. The PNN network produces an accuracy rate of $90.2 \%$. The study and development of PNN, LVQ Neural Network and BPN for classification of fatty and cirrhosis liver diseases is reported in [15]. Results show that, biorthogonal wavelet based statistical features were producing better results compared to the statistical features extracted directly from an image without applying wavelet transform.

A computer-aided classification system is developed for the analysis of benign and malignant liver tumors from CT images by means of curvelet transform [16] and neural network. HCC and cavernous hemangioma are the two kinds of liver diseases were identified. The categorization accuracy of tumors using curvelet based texture feature extraction is $94.3 \%$ whereas it is just $88.6 \%$ using wavelet based method. The accuracy rates of curvelet-based tumor classification have significantly higher. Fast Discrete Curvelet Transform (FDCT) is introduced [17] to train and classify the liver tumor into hemangioma and hepatoma. The mean, standard deviation, energy and entropy were identified for every sub bands and set in the feature vector. The input dataset consists of 90 images which are clustered into training and a testing set with 45 datasets each. The classification accuracy of tumors using FDCT based texture feature extraction is $93.3 \%$. But it is only $88.9 \%$ using wavelet based method. FDCT based texture features considerably improve the classification rate of liver tumors.

The co-occurrence matrices are used to extract feature like contrast, correlation, entropy, homogeneity and energy. Then the PNN is trained using extracted features to classify liver tumors as malignant and benign. [18] The proposed system was tested and it produces accuracy of 93.75\%. The knowledge base is used for medical image diagnosis [19]. Revised GMDH-type neural network algorithm can recognize the characteristics of the medical images correctly using feedback loop computation. The Liver CAD system is developed using Contourlet Transform [20] for feature extraction to classify tumors automatically in the liver. HCC and hemangioma are identified. The performance of the CAD system indicates that the sensitivity and specificity of the proposed system are $97.1 \%$ and $91.4 \%$ respectively, compared to the mean sensitivity and specificity of $91.4 \%$ and $85.7 \%$ obtained by feature extraction using Wavelet Transforms.

An automatic classification system for early recognition of liver diseases from CT images was proposed. In feature extraction biorthogonal wavelet, Gray-level cooccurrence matrix and FDCT techniques are used [21]. The textual information obtained was used to train various neural networks such as BPN, PNN and Cascade feed forward BPN (CFBPN). Totally 40 hepatoma and 30 hemangioma images are considered for training and testing. The kurtosis, skewness, energy, information correlation, inverse difference moment, entropy feature combinations performed better in BPN, PNN and CFBPN neural networks with $96 \%$. Gray scale co-occurrence matrix is extracted from tumor image and extracted features are fed as input to every $\mathrm{NN}$. It is performed well in BPN with accuracy $96 \%$. PNN and CFBPN accuracy results are less when compared with BPN. Automatic lesion segmentation, texture feature extraction and classification of malignant and benign tumors is proposed [22]. Both first order statistic and second order statistic features are extracted from the gray level and contourlet detail coefficients. The extracted feature sets are classified by a PNN classifier into benign and malignant. Results indicate that the contourlet coefficient texture is effective for classifying malignant and benign liver tumors.

\subsection{Multi Level Neural Network}

A classifier consisting of three sequentially placed neural networks for four classes of hepatic tissues is developed [23]. 147 samples were used, 76 of which belong to healthy ones, 19 to cysts, 28 to hemangioma and 24 to HCC. Eight cooccurrence texture features namely angular second moments, contrast, correlation, sum of squares, inverse difference moment, entropy, homogeneity, cluster tendency are calculated for six different values of the pixel spacing. Regions of interest (ROIs) are taken from CT images of normal liver, hepatic cysts, hemangioma, and HCC. The 48 textural features are extracted from the SGLDM [24] which are obtained from ROI. The SFS, the sequential floating forward selection, and a GA has been applied to each individual $\mathrm{NN}$ for feature selection. Three sequentially placed feed-forward neural networks are used for classification. NN classifiers are proposed for classifying CT liver images into normal, hepatic cyst, hemangioma, and HCC. FOS, SGLDM, GLDS, TEM and FDM [25] were used for feature extraction from ROI. Dimensionality of feature vector was reduced using GA Classification is then carried out by a system of four NNs, each using as input one of the above feature sets. The members of the NN system are 4-class NNs trained by the back propagation algorithm with adaptive learning rate and 
momentum. The final result of the system is based on the application of a voting method across the output of the individual NNs. The manifold classification scheme by means of the five sets of texture features results in considerably improved performance

A CAD system architecture is developed [26] which are able to accurately classify hepatic as normal liver, hepatic cyst, hemangioma, and HCC. For each CT liver ROI, five types of texture feature sets, based on FOS, SGLDM, GLDS, TEM and FDM were extracted. GA-based feature selection method was applied for feature selection. First Classifier consists of five multilayer perceptron neural networks (NNs). The input of each classifier is one of the calculated texture feature sets or its condensed version after GA based feature selection. The second Classifier consists of five different primary classifiers, namely one multilayer perceptron $\mathrm{NN}$, one PNN, and three k-nearest neighbor classifiers, each fed with the combination of the five texture feature sets or with their reduced versions. Appropriate voting schemes were used in the final decision of each classifier.

\subsection{Minimum Distance Classifier}

Zernike moments and Legendre moments are utilized for classification of normal and HCC liver using CT images [27]. 200 ROI were used out of which 140 belong to the healthy liver class and 60 to HCC. Each ROI was segmented into multiple $8 \times 8$ segments out of which 75 were used for training and the left over for testing. Statistical feature selection method based on't'-test was proposed [28] to identify the moment orders. They worked with fifty scans of healthy liver and seventy scans of abnormal liver. The Zernike moment features are effective in distinguishing the soft liver tissues in normal and abnormal. The nearest mean classifier is computed based on Euclidean distance measure. The classification accuracy rate with Zernike feature vector for normal liver is $98.33 \%$ and that of liver with HCC is $90.67 \%$ and Legendre feature vector yielded $97.66 \%$ correct classification for normal liver and $81.67 \%$ of liver with HCC

\section{PERFORMANCE SUMMARY OF TEXTURAL ANALYSIS OF METHODS}

Today's liver CAD systems cannot detect $100 \%$ of pathological changes. An accurate hit is termed a True Positive (TP), while the wrong marking on the healthy part constitute a False Positive (FP). The less FPs point to, the higher the specificity is. A low specificity lessens the acceptance of the CAD system because the user has to identify all of these wrong hits. The performance of the CAD system is evaluated using various parameters like accuracy, sensitivity, specificity, PPV (Positive predictive value) and NPV (Negative predictive value). CAD systems seek to highlight suspicious structures. Performance summary of various texture methods to characterize liver diseases is given in Table 1.

\subsection{Discussion}

In diagnosing liver diseases, CT has become one of the major imaging modalities. Different authors have used different techniques for classification of liver diseases from CT. A summary of classification results using different textural methods can be of significant value in identifying future work. While comparing all methods with the help of accuracy measure, the experimental results of the method proposed by Gletsos (2001) achieved the accuracy of $98 \%$ to classify the Normal liver, hepatic cysts, Hemangioma, and HCC. High performance is achieved by the suitability of co-occurrence texture features, the superiority of GAs for feature selection, and the Multi level Neural Network classifiers. Many authors have shown SVM to be an efficient method for classification problems. The method proposed by Wang et al (2009) shows the total accuracy rate of $97.78 \%$ with the multiclass SVM. Kumar et al (2010, 2011, and 2012) reveals that curvlet and contourlet transforms performs better than wavelet transform for classification. Using curvlet and contourlet transform he obtained the classification rate of about $94.3 \%$. Mala et al (2010) and Gunasundari et al (2012) concluded that the performance of PNN is good when it is compared with other neural networks. The Bharathi et al (2008) used orthogonal moments to classify the liver diseases from abdominal CT. The experimental results show that the proposed orthogonal moments like Zernike moments and Legendre moments features are effective texture descriptors. Fig1 depicts the disease name and number of authors worked on that disease. The horizontal axis shows disease names used in different classification schemes whereas vertical axis represents the number of authors identified the diseases from abdominal CT. Only some diseases like HCC and Hemangioma are considered by many authors. Diseases like Fatty liver, cirrhosis and other diseases are not focused by many authors. Many authors have addressed only classification of liver diseases with free hand ROI. They didn't work with automatic segmentation. Also they have identified diseases only from 2D abdominal CT.

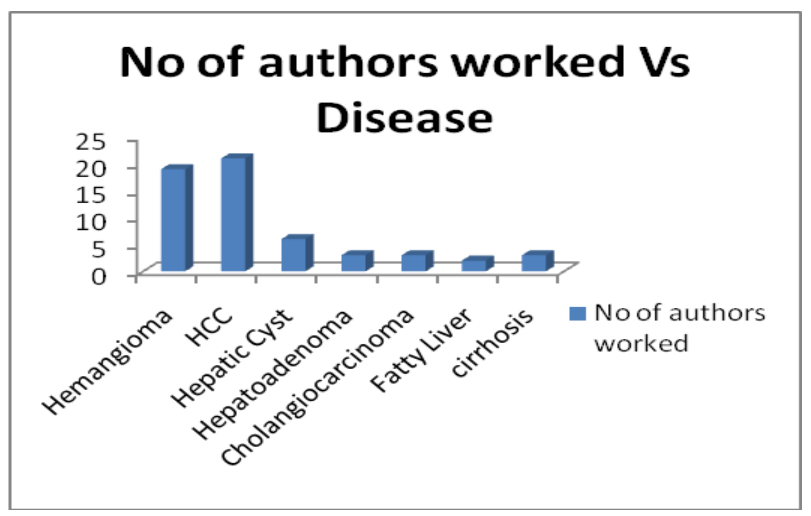

Fig 1: No of Authors worked Vs Disease Name

\section{CONCLUSION AND FUTURE WORK}

With the rapid development of science and technology, computer doctors may be able to diagnose almost all diseases. There has been a lot of development in the scope, technique, and ability of Liver CAD systems. In this study we have presented the existing methods for classifying liver diseases from the abdominal CT. Hence it is concluded that the neural networks, Genetic algorithm and conventional image processing operations can be successfully used for liver disease diagnosis from abdominal CT. This research can further be extended in many directions. First, existing techniques can be applied to classify other diseases. Second, more texture features and techniques can be used to improve the performance, Third increase data sets to improve the system robustness and finally all texture analysis methods using the same data set and similar performance measures can be studied to provide better comparison. We have discussed only the textural techniques for the classification of liver diseases from abdominal CT. It can also be done in depth for other types of medical images like MRI. 


\begin{tabular}{|c|c|c|c|c|}
\hline $\begin{array}{l}\text { References/ } \\
\text { Author Name }\end{array}$ & Textural Features & Classifier & Disease & Accuracy \\
\hline $\begin{array}{l}{[8]} \\
\text { Wang et al (2009) }\end{array}$ & $\begin{array}{l}\text { FOS, SGLDM, GLRLM and } \\
\text { GLDM }\end{array}$ & $\begin{array}{l}\text { Multi Class } \\
\text { SVM }\end{array}$ & $\begin{array}{l}\text { HCC, Hemangioma, } \\
\text { Normal }\end{array}$ & $\mathbf{9 7 . 7 8} \%$ \\
\hline $\begin{array}{l}{[13]} \\
\text { Mala et al (2006 a) }\end{array}$ & $\begin{array}{l}\text { Biorthogonal wavelet transform - } \\
\text { Angular second } \\
\text { moment or Energy, Contrast, } \\
\text { Homogeneity and Entropy }\end{array}$ & LVQ & $\begin{array}{l}\text { Benign(Hemangiom } \\
\text { a and Adenoma) and } \\
\text { Malignant (HCC, } \\
\text { Cholangio } \\
\text { carcinoma) }\end{array}$ & $92 \%$ \\
\hline $\begin{array}{l}{[15]} \\
\text { Mala et al (2010) }\end{array}$ & $\begin{array}{l}\text { Biorthogonal wavelet-based } \\
\text { statistical texture feature } \\
\text { Feature Selection: } \\
\text { Sequential Forward Floating } \\
\text { Search (SFFS) and GA }\end{array}$ & $\begin{array}{l}\text { PNN, LVQ, } \\
\text { BPN }\end{array}$ & $\begin{array}{l}\text { Fatty Liver and } \\
\text { cirrhosis }\end{array}$ & $\begin{array}{l}\text { PNN }-96 \% \\
\text { LVQ }-93 \% \\
\text { BPN }-80\end{array}$ \\
\hline $\begin{array}{l}{[17]} \\
\text { Kumar et al }(2010 \text { b) }\end{array}$ & $\begin{array}{l}\text { Fast Discrete Curvelet Transform } \\
\text { (FDCT) }\end{array}$ & $\begin{array}{l}\text { Feed Forward } \\
\text { NN }\end{array}$ & $\begin{array}{l}\text { HCC and cavernous } \\
\text { Hemangioma }\end{array}$ & $\begin{array}{l}\text { Curvelet - } \\
93.3 \% \\
\text { Wavelet - } \\
88.9 \%\end{array}$ \\
\hline $\begin{array}{l}\text { [21] } \\
\text { Gunasundari et al } \\
2012\end{array}$ & $\begin{array}{l}\text { Co-occurrence matrix and Fast } \\
\text { discrete Curvelet transform }\end{array}$ & $\begin{array}{l}\text { BPN, PNN } \\
\text { and Cascade } \\
\text { feed forward } \\
\text { BPN } \\
\text { (CFBPN). }\end{array}$ & $\begin{array}{l}\text { Hemangioma, and } \\
\text { HCC }\end{array}$ & $\begin{array}{l}\text { BPN }-96 \% \\
\text { PPN }-96 \% \\
\text { CFBPN }-96 \%\end{array}$ \\
\hline $\begin{array}{l}{[22]} \\
\text { Kumar et al (2012) }\end{array}$ & $\begin{array}{l}\text { Gray-Level first-order statistics } \\
\text { (GLFOS), Gray level co- } \\
\text { occurrence matrix, Contourlet } \\
\text { coefficient first-order statistics } \\
\text { (CCFOS), Contourlet coefficient } \\
\text { co- occurrence matrices (CCCMs) } \\
\text { Feature Selection : PCA }\end{array}$ & PNN & $\begin{array}{l}\mathrm{HCC} \text { and } \\
\text { Hemangioma }\end{array}$ & $\begin{array}{l}\text { FOS - } 79 \\
\text { GLCM }-86 \\
\text { CCFOS }-93 \\
\text { CCCM }-94\end{array}$ \\
\hline $\begin{array}{l}{[23]} \\
\text { Gletsos et al. (2001) }\end{array}$ & $\begin{array}{l}\text { Angular second moment, contrast, } \\
\text { correlation, sum of squares, inverse } \\
\text { difference moment, entropy, } \\
\text { homogeneity, cluster tendency } \\
\text { Feature selection : sequential } \\
\text { forward floating selection (SFFS) }\end{array}$ & $\begin{array}{l}\text { Feed-Forward } \\
\text { NN }\end{array}$ & $\begin{array}{l}\text { Normal liver, } \\
\text { hepatic cysts, } \\
\text { Hemangioma, and } \\
\text { HCC }\end{array}$ & $98 \%$ \\
\hline $\begin{array}{l}{[24]} \\
\text { Gletsos et al (2003) }\end{array}$ & $\begin{array}{l}48 \text { texture characteristics from } \\
\text { spatial gray-level co-occurrence } \\
\text { matrices- Angular Second } \\
\text { Moment, Contrast, correlation, sum } \\
\text { of Square - Variance, Inverse } \\
\text { Difference Moment, Entropy, } \\
\text { Homogeneity, Cluster Tendency } \\
\text { Feature Selection: } \\
\text { SFS, SFFS, GA }\end{array}$ & $\begin{array}{l}\text { Feed-forward } \\
\text { NN }\end{array}$ & $\begin{array}{l}\text { Normal liver, } \\
\text { hepatic cysts, } \\
\text { Hemangioma, and } \\
\text { HCC }\end{array}$ & $97 \%$ \\
\hline $\begin{array}{l}25] \\
\text { Mougiakakou et } \\
\text { al.(2003) }\end{array}$ & $\begin{array}{l}\text { FOS, SGLDM, } \\
\text { GLDM, TEM, and } \\
\text { FDM } \\
\text { Feature Selection : GA }\end{array}$ & $\begin{array}{l}\text { 4-class NN- } \\
\text { Back } \\
\text { propagation } \\
\text { Algorithm }\end{array}$ & $\begin{array}{l}\text { Normal, Hepatic } \\
\text { cyst, Hemangioma, } \\
\text { and HCC. }\end{array}$ & $97 \%$ \\
\hline $\begin{array}{l}\text { [26] } \\
\text { Mougiakakou et al } \\
(2007)\end{array}$ & $\begin{array}{l}\text { FOS, SGLDM, } \\
\text { GLDM, TEM, and } \\
\text { FDM } \\
\text { Feature Selection : GA }\end{array}$ & $\begin{array}{l}5 \text { Multilayer } \\
\text { Perceptron NN }\end{array}$ & $\begin{array}{l}\text { Normal liver, } \\
\text { Hepatic cyst, } \\
\text { Hemangioma, and } \\
\text { HCC }\end{array}$ & $84.96 \%$ \\
\hline
\end{tabular}

Table 1: Summary of some techniques to identify Liver Diseases from Abdominal CT 


\section{REFERENCES}

[1] Srinivasan GN, and Shobha G. (2008) Statistical Texture Analysis. In: Proc. of World Academy of Science, Engineering And Technology 36: 1264-1269

[2] Haralick RM, Shanmugam K, Dinstein I (1973) Texture features for image classification. IEEE Transaction on System, Man and Cybernetics SMC-3 (6) : 610-621

[3] Rathore S, Iftikhar MA, Hussain M, Jalil A (2011) Texture analysis for liver segmentation and classification: a survey. In: Proc. of International Conference of IEEE on Frontiers of Information Technology 121-126

[4] Huang, YL, Chen JH, Shen WC (2006) Diagnosis of Hepatic Tumors with Texture Analysis in Non enhanced Computed Tomography Images. Acad Radiol 2006 13:713-720

[5] Lee CC, Chiang YC, Tsai CL, Chen SH (2007 a) Distinction of Liver Disease from CT images using Kernel-based Classifiers. ICMED, 1:2:113-120

[6] Lee CC, Chen SH, Chiang YC (2007 b) Classification of Liver Disease from CT Images Using a Support Vector Machine. Journal of Advanced Computational Intelligence and Intelligent Informatics 11:4: 396-402

[7] Nawaz S (2008) Hepatic lesions classification by ensemble of SVMs using statistical features based on cooccurrence matrix. In: Proc. of International Conference of IEEE on Emerging Technologies 21-26

[8] Wang L, Zhang Z, Liu J, Jiang B, Duan X, Xie Q, Hu D, Li Z (2009) Classification of Hepatic Tissues from CT Images Based on Texture Features and Multiclass Support Vector Machines. In: Proc of International Symposium on Neural Netwroks: Advances in Neural Network

[9] Mir AH, Hanmandlu M, Tandon SN (1995) Texture analysis of CT images. IEEE Engineering in Medicine and Biology 14: 6:781-786

[10] Chen EL, Chung PC, Chen CL, Tsai HM, Chang CI (1998) An Automatic Diagnostic System for CT Liver Image Classification. IEEE Transactions on Biomedical Engineering 45:6:783-794

[11] Valavanis I, Mougiakakou SG, Nikita KS, Nikita A (2004) Computer Aided Diagnosis of CT Focal Liver Lesions by an Ensemble of Neural Network and Statistical Classifier. In: Proc. of International Conference of IEEE on Neural Networks 3:1929-1934

[12] Mala K, Sadasivam V (2005) Automatic segmentation and classification of diffused liver diseases using wavelet based texture analysis and Neural Network. In Proc of International Conference of IEEE on INDICON 216-219

[13] Mala K, Sadasivam V (2006 a) Wavelet based texture analysis of Liver tumor from Computed Tomography images for characterization using Linear Vector Quantization Neural Network. In: Proc. Of International conference of IEEE on Advanced Computing and Communication 267 - 270

[14] Mala K, Sadasivam V, S.Alagappan (2006 b) Neural Network based Texture Analysis of Liver Tumor from Computed Tomography Images. International Journal of Biological and Life Sciences 2:1:33-40

[15] Mala K , Sadasivam S (2010) Classification of Fatty and Cirrhosis Liver Using Wavelet-Based Statistical Texture Features and Neural Network Classifier. International Journal of Software Informatics 4:2:151-163
[16] Kumar SS, Moni RS (2010 a) Diagnosis of Liver Tumor from CT Images using Curvelet Transform. International Journal on Computer Science and Engineering 2:4: 11731178

[17] Kumar SS, Moni RS (2010 b) Diagnosis of Liver Tumor from CT Images using Curvelet Transform. International Journal of Computer Application Special Issue on CASCT 1:1-6

[18] Lakshmana B, Arakeri MP (2011) Texture based characterization of liver tumor on computed tomography images. In: Proc. of the International Conference \& Workshop on Emerging Trends in Technology Pages 141-144

[19] Kondo T, Ueno J, Takao S (2011) Hybrid GMDH-type neural network using artificial intelligence and its application to medical image diagnosis of liver cancer. In: Proc. Of International Symposium of IEEE on System Integration 1101-1106

[20] Kumar SS, Moni RS, Rajeesh J (2011) Contourlet Transform Based Computer-Aided Diagnosis System for Liver Tumors on Computed Tomography Images. In: Proc. of International Conference of IEEE on Signal Processing, Communication, Computing and Networking Technologies 217 - 222

[21] Gunasundari S, Suganya Ananthi M (2012) Comparison and Evaluation of Methods for Liver Tumor Classification from CT Datasets. International Journal of Computer Applications 39:18:46 -51

[22] Kumar SS, Moni RS, Rajeesh J (2012) Liver tumor diagnosis by gray level and contourlet coefficients texture analysis. International Conference on Computing, Electronics and Electrical Technologies 557 $-562$

[23] Glestos M, Mougiakakou SG, Matsopoulos GK., Nikita KS, Nikita, AS, Kelekis D (2001) Classification of hepatic lesions from CT images using texture features and neural networks. In: Proc. Of 23rd Annual EMBS International Conference of IEEE on Engineering in Medicine and Biology Society 3: 2748-2752

[24] Gletsos M, Mougiakakou SG, Matsopoulos GK, Nikita KS, Nikita AS, Kelekis D (2003) A Computer-Aided Diagnostic System to Characterize CT Focal Liver Lesions: Design and Optimization of a Neural Network Classifier. IEEE Transactions on Information Technology in Biomedicine 7:3:153 - 162

[25] Mougiakakou SG, Valavanis I, Nikita KS, Nikita A, Kelekis D (2003) Characterization of CT liver lesions based on texture features and a multiple Neural Network classification scheme. In: Proc of lntemational Conference of IEEE on Engineering in Medicine and Biology Society, 2:1287-1290

[26] Mougiakakou SG, Valavanis I, Nikita A, Nikita KS (2007) Differential diagnosis of CT focal liver lesions using texture features, feature selection and ensemble driven classifiers. Artificial Intelligence in Medicine 41:25-37

[27] Bharathi VS, Vijilious MAL, Ganesan L (2007) Orthogonal Moments based texture analysis of CT liver images. In: Proc. Of International Conference of IEEE on Computational Intelligence and Multimedia Applications 2:249-253

[28] Bharathi VS, Ganesan L (2008) Orthogonal moments based texture analysis of CT liver images. Pattern Recognition Letters 29:1868-1872 